\title{
Nonexistence of Global Weak Solutions of Nonlinear Keldysh Type Equation with One Derivative Term
}

\author{
Kangqun Zhang (iD \\ Department of Mathematics and Physics, Nanjing Institute of Technology, Nanjing 211167, China \\ Correspondence should be addressed to Kangqun Zhang; chkqnju@hotmail.com
}

Received 28 April 2018; Revised 17 June 2018; Accepted 16 July 2018; Published 1 August 2018

Academic Editor: Ming Mei

Copyright (C) 2018 Kangqun Zhang. This is an open access article distributed under the Creative Commons Attribution License, which permits unrestricted use, distribution, and reproduction in any medium, provided the original work is properly cited.

We focus on the nonexistence of global weak solutions of nonlinear Keldysh type equation with one derivative term. In terms of the analysis of the first Fourier coefficient, we show the solution of singular initial value problem and singular initial-boundary value problem of the nonlinear equation with positive initial data blow-up in some finite time interval.

\section{Introduction}

In this paper we consider the nonexistence of global weak solution of nonlinear Keldysh type equation (1) with modified initial data (2) and boundary value (3); that is,

$$
\begin{aligned}
t \partial_{t}^{2} u-\triangle u+b \partial_{t} u & =f(u), \quad \text { in }(0, T) \times \Omega \\
u(0, x) & =\varphi_{1}(x), \\
\lim _{t \rightarrow 0} t^{b} \partial_{t} u(t, x) & =\varphi_{2}(x)
\end{aligned}
$$

in $\Omega$

and

$$
u(t, x)=0 \quad \text { on }(0, T) \times \partial \Omega
$$

where $\triangle=\sum_{i=1}^{n} \partial_{x_{i}}^{2}$ is the Laplace differential operator and $b \in(0,1)$ is a parameter. Here (1)-(2) is a singular initial value problem for $\Omega=\mathbb{R}^{n}$. And (1)-(3) is a singular initialboundary value problem when $\Omega \subseteq \mathbb{R}^{n}$ is a bounded domain with a piecewise smooth boundary $\partial \Omega$. The singularity of the given second datum was analyzed by T. Hristov in [1]. In order to formulate the mechanism of blow-up, we assume that, for $s>s_{0}$ with a positive constant $s_{0}$, the nonlinear source term $f(s)$ is a convex function such that

$$
f(s)-\mu s \geq c s^{1+p}, \quad p>0
$$

with some positive constants $\mu$ and $c$.
The Keldysh type equations are known in some specific applications in plasma physics, optics, and analysis on projective spaces [2]. In one-dimensional space, homogeneous equation of (1) turns into the classic Keldysh equation which is closely connected with flow dynamics $[3,4]$. In this case, Chen S.X. [5] established the fundamental solutions to the corresponding differential operator for $b<1 / 2$ by using the finite part of divergence integrals in theory of distribution. For $n=3$, the existence of singular solution to Protter problem and Protter-Morawetz problem was considered in $[6,7]$.

In the upper half space, (1) is a hyperbolic equation with variable propagation speed and characteristics degenerate on $t=0$. For nonlinear wave equation with constant coefficients, there exist many well-known results including existence and blow-up of solutions. For the existence of solutions, one can see [8-11]. Here we emphasize the nonexistence of global solution; then we will enumerate some classical results. In [12] for $n=3$, Keller used a comparison theorem and Riemann function showed the solution is blow-up in finite time. In [13], Jörgens extended Keller's idea to the case for nonlinear wave equation with $n>3$. For more results, one can see [1418]. Similar idea of the proof also has been used to establish the blow-up of solution of nonlinear parabolic equations by Kaplan [19] and Fujita [20, 21].

In this paper, we use the first Fourier coefficient of the solution to show the blow-up of solution. This method was first introduced by Kaplan [19] to study a nonlinear secondorder parabolic equation, later modified by Glassey [14] for 
classical solutions of nonlinear wave equations and extended by Levine $[22,23]$ to weak solutions of parabolic and wave equations. In order to show the first Fourier coefficient of the solution is positive and approaches infinity at finite time, we derive a nonlinear second-order singular differential inequality:

$$
\frac{d}{d t}\left(t^{b} F^{\prime}(t)\right) \geq c t^{b-1} F^{1+p}(t)
$$

Then, based on the cautious calculation of singular differential inequality (5), we can deal with the nonexistence of global solution to the nonlinear problem and the proof is independent of the weak conservation of energy law and concavity of argument.

This paper is organized as follows: In Section 2, the blow-up result for singular initial-boundary value problem is obtained. In Section 3, based on a similar method used in Section 2 but with some differences in calculus, we show the blow-up of solution to singular initial value problem.

\section{Singular Initial-Boundary Value Problem}

In this section, we consider the blow-up of solution of problem (1)-(3) on a bounded domain $\Omega$. The method of proof is independent of the spatial dimension and the Riemann function.

Let $\psi(x)$ denote the first eigenfunction of Dirichlet problem:

$$
\begin{aligned}
\triangle \psi+\lambda \psi=0, & \text { in } \Omega \\
\psi(x)=0 & \text { on } \partial \Omega
\end{aligned}
$$

with the first eigenvalue $\lambda$. According to a classical result ([24], Vol.I, pp. 451-455), then there is only one normalized eigenfunction $\psi(x)>0$ satisfying

$$
\int_{\Omega} \psi(x) d x=1
$$

Now we consider problem (1)-(3) in bounded domain $\Omega$ with smooth boundary $\partial \Omega$.

Assume that

(a) $\varphi_{1}(x) \geq 0, \varphi_{2}(x) \geq 0$ for all $x \in \Omega$; there exist two points $x_{i} \in \Omega, i=1,2$, such that $\varphi_{1}\left(x_{1}\right)>0, \varphi_{2}\left(x_{2}\right)>$ 0 ;

(b) $\int_{\Omega} \psi(x) \varphi_{1}(x) d x=\alpha>0, \int_{\Omega} \psi(x) \varphi_{2}(x) d x=\beta>0$.

Set

$$
(\phi(t, \cdot), u(t, \cdot))=\int_{\Omega} \phi(t, x) u(t, x) d x
$$

Definition 1. The function $u(t, x)$ is called a weak solution of initial-boundary value problem (1)-(3) if $u(t, \cdot)$ and $t^{b} \partial_{t} u(t, \cdot)$ are continuous with respect to $t$, if u satisfies (2) and (3), and if for all $\phi:(0, T) \times \Omega \longrightarrow \mathbb{R}$ which are twice continuously differentiable and satisfies

$$
\begin{aligned}
& t\left(\phi(t, \cdot), \partial_{t} u(t, \cdot)\right)=\int_{0}^{t}\left[(1-b)\left(\phi(\tau, \cdot), \partial_{\tau} u(\tau, \cdot)\right)\right. \\
& \quad+\tau\left(\partial_{\tau} \phi(\tau, \cdot), \partial_{\tau} u(\tau, \cdot)\right)+(\Delta \phi(\tau, \cdot), u(\tau, \cdot)) \\
& \quad+(\phi(\tau, \cdot), f(u(\tau, \cdot)))] d \tau .
\end{aligned}
$$

Theorem 2. Assume that a weak solution of problem (1)-(2) with $\mu \geq \lambda$ in (4) under assumptions (a)-(b) in the sense of Definition 1 exists; then there is some positive constant $T$ such that

$$
\begin{aligned}
& \lim _{t \longrightarrow T^{-}}\|u(t, \cdot)\|_{L^{\infty}(\Omega)}=+\infty, \\
& \lim _{t \rightarrow T^{-}}\|u(t, \cdot)\|_{L^{q}(\Omega)}=+\infty, \quad q \in[1,+\infty) .
\end{aligned}
$$

Proof. For $\psi(x)$ defined in (6)-(7), set

$$
F(t)=(\psi(x), u(t, x))=\int_{\Omega} \psi(x) u(t, x) d x,
$$

and then $F(0)=\alpha>0$ and

$$
F^{\prime}(t)=\left(\psi(x), \partial_{t} u(t, x)\right)=\int_{\Omega} \psi(x) \partial_{t} u(t, x) d x
$$

with $\lim _{t \rightarrow 0} t^{b} F^{\prime}(t)=\beta>0$. Since there is no differentiability of second order on the weak solution $u$ with respect to $t$ in Definition 1, then we will derive the second order of $F(t)$ by use of (9). Multiplying $t$ on (13), in terms of (8) and (9), we obtain

$$
\begin{aligned}
& F^{\prime \prime}(t)=\left(t^{-1} t F^{\prime}(t)\right)^{\prime}=-t^{-1} F^{\prime}(t)+t^{-1}\left(t F^{\prime}(t)\right)^{\prime} \\
& =t^{-1}\left[-b\left(\psi(x), \partial_{t} u(t, x)\right)+(\psi(x), f(u(t, x)))\right. \\
& \quad-\lambda(\psi(x), u(t, x))] .
\end{aligned}
$$

According to the convexity of $f(\cdot)$ and Jensen's inequality, one has

$$
\begin{aligned}
(\psi(x), f(u(t, x))) & \geq f((\psi(x), u(t, x))) \\
& =f(F(t)) .
\end{aligned}
$$

Then substitute (15) into (14), together with (4) for $\lambda=\mu$; we obtain

$$
\begin{aligned}
& F^{\prime \prime}(t) \geq t^{-1}\left[-b\left(\psi(x), \partial_{t} u(t, x)\right)\right. \\
& \quad+f(\psi(x), u(t, x))-\lambda(\psi(x), u(t, x))] \\
& \quad \geq-b t^{-1} F^{\prime}(t)+c t^{-1} F^{1+p}(t)
\end{aligned}
$$

which is equivalent to

$$
\frac{d}{d t}\left(t^{b} F^{\prime}(t)\right) \geq c t^{b-1} F^{1+p}
$$

Note that $F(0)=\alpha>0$ and $\lim _{t \rightarrow 0} t^{b} F^{\prime}(t)=\beta>0$; then there exists a positive number $\eta$ such that $F(t)>\alpha$ for $t \epsilon$ 
$(0, \eta)$. Hence $(17)$ means that $t^{b} F^{\prime}(t)$ is strictly increasing on the interval $(0, \eta)$. Multiply $t^{b} F^{\prime}(t)$ on (15) and then integrate it over $(\varepsilon, t)$ such that $(\varepsilon, t) \in(0, \eta)$,

$$
\begin{aligned}
\left(t^{b} F^{\prime}(t)\right)^{2} \geq & \left(\varepsilon^{b} F^{\prime}(\varepsilon)\right)^{2} \\
& +2 c \int_{\varepsilon}^{t} \tau^{2 b-1} F^{1+p}(\tau) F^{\prime}(\tau) d \tau .
\end{aligned}
$$

It follows that $F^{\prime}(t)>\beta t^{-b}$ and $F(t)>\alpha$ on the entire existence interval $(0, \eta)$.

Now, for $\varepsilon>0$, we set

$$
\begin{aligned}
& H(t)=\int_{\varepsilon}^{t} \tau^{2 b-1} F^{1+p}(\tau) F^{\prime}(\tau) d \tau, \\
& G(t)=(2+p)^{-1} F^{2+p}(t) .
\end{aligned}
$$

On one hand, it is easy to verify that $H^{\prime}(t)=t^{2 b-1} G^{\prime}(t)$ holds and $H(t)>0, G(t)>0$. For $b \geq 1 / 2$, integrating by parts, it follows that

$$
\begin{aligned}
H(t) \leq & t^{2 b-1} G(t) \\
& -\left(\varepsilon^{2 b-1} G(\varepsilon)+(2 b-1) \int_{\varepsilon}^{t} \tau^{2(b-1)} G(\tau) d \tau\right) \\
\leq & t^{2 b-1} G(t) .
\end{aligned}
$$

On the other hand, in terms of (17)-(20), we derive that

$$
\begin{gathered}
\left(H^{\prime}(t)\right)^{2}=t^{2 b-2} F^{2+2 p}(t)\left(t^{b} F^{\prime}(t)\right)^{2} \geq(2+p)^{2+2 p} \\
\cdot t^{2(b-1)}\left(\left(\varepsilon^{b} F^{\prime}(\varepsilon)\right)^{2}+2 c H(t)\right) G^{1+p /(2+p)}(t) .
\end{gathered}
$$

Then, substituting (21) into (22), we obtain

$$
\begin{aligned}
& \left(H^{\prime}(t)\right)^{2} \geq(2+p)^{2+2 p} \\
& \cdot t^{-1+p(1-2 b) /(2+p)}\left(\left(\varepsilon^{b} F^{\prime}(\varepsilon)\right)^{2}+2 c H(t)\right) \\
& \cdot H^{1+p /(2+p)}(t),
\end{aligned}
$$

which is equivalent to

$$
\begin{aligned}
& {\left[\left(\left(\varepsilon^{b} F^{\prime}(\varepsilon)\right)^{2}+2 c H(t)\right) H^{1+p /(2+p)}(t)\right]^{-1 / 2} H^{\prime}(t)} \\
& \quad \geq(2+p)^{1+p} t^{-1 / 2+p(1-2 b) / 2(2+p)}
\end{aligned}
$$

For $b \in(1 / 2,1)$, integrate inequality $(24)$ over $(\varepsilon, t)$; then

$$
\begin{gathered}
+\infty>\int_{H(\varepsilon)}^{H(t)}\left(\left(\varepsilon^{b} F^{\prime}(\varepsilon)\right)^{2}+2 c H(\tau)\right)^{-1 / 2} \\
\cdot H^{-1 / 2-p / 2(2+p)}(\tau) d H(\tau) \geq \int_{\varepsilon}^{t}(2+p)^{1+p} \\
\cdot \tau^{-1 / 2+p(1-2 b) / 2(2+p)} d \tau \geq\left(\frac{1}{2}+\frac{p(1-2 b)}{2(2+p)}\right)^{-1}(2 \\
+p)^{1+p}\left(t^{1 / 2+p(1-2 b) / 2(2+p)}-\varepsilon^{1 / 2+p(1-2 b) / 2(2+p)}\right) .
\end{gathered}
$$

Fix $\varepsilon$; inequality (25) implies that there is a singularity of $H(t)$ at some point $T \leq T_{0}$, where

$$
\begin{aligned}
T_{0} & =\left[\varepsilon^{1 / 2+p(1-2 b) / 2(2+p)}+\left(\frac{1}{2}+\frac{p(1-2 b)}{2(2+p)}\right)(2\right. \\
& +p)^{-1-p} \times \int_{H(\varepsilon)}^{+\infty}\left(\left(\varepsilon^{b} F^{\prime}(\varepsilon)\right)^{2}+2 c t\right)^{-1 / 2} \\
& \left.\cdot t^{-1 / 2-p / 2(2+p)} d t\right]^{1 /(1 / 2+p(1-2 b) / 2(2+p))} .
\end{aligned}
$$

Then, for some $t_{0} \leq T_{0}$, we derive

$$
\lim _{t \rightarrow T^{-}} H(t)=+\infty
$$

Moreover, by a direct computation on (21), we have

$$
\lim _{t \rightarrow T^{-}} H(t) \leq T^{2 b-1} \lim _{t \rightarrow T^{-}} G(t) .
$$

Hence, combining (20) through (27) and (28), we confirm that

$$
\lim _{t \longrightarrow T^{-}} F(t)=+\infty
$$

Finally, because of $\psi(x)>0$, in terms of Hölder's inequality, we have

$$
\begin{aligned}
F(t) & =|F(t)|=|(\psi(x), u(t, x))| \\
& =\|\psi(x) u(t, x)\|_{L^{1}(\Omega)} \\
& \leq\|\psi(x)\|_{L^{p}(\Omega)}\|u(t, x)\|_{L^{q}(\Omega)}
\end{aligned}
$$

which implies (11). In particular, since $\psi(x)$ satisfies (7), then, from (30), we obtain

$$
F(t)<\|u(t, x)\|_{L^{\infty}(\Omega)} .
$$

This derives (10)

Next, we consider the case for $b \in[0,1 / 2)$. Therefore $2 b-$ $1<0$. Then, for $0<\varepsilon \leq \tau \leq t$, we have $t^{2 b-1} \leq \tau^{2 b-1} \leq \varepsilon^{2 b-1}$. Since $c>0, p>0, F(t)>0$, and $F^{\prime}(t)>0$ on the entire existence interval from (18), it follows that

$$
\begin{aligned}
& \left(t^{b} F^{\prime}(t)\right)^{2} \\
& \quad \geq t^{2 b-1}\left[\varepsilon\left(F^{\prime}(\varepsilon)\right)^{2}+2 c \int_{\varepsilon}^{t} F^{1+p}(\tau) F^{\prime}(\tau) d \tau\right] .
\end{aligned}
$$

This is equivalent to

$$
\begin{aligned}
& \left(F^{\prime}(t)\right)^{2} \\
& \quad \geq t^{-1}\left[\varepsilon\left(F^{\prime}(\varepsilon)\right)^{2}+\frac{2 c}{2+p}\left(F^{2+p}(t)-F^{2+p}(\varepsilon)\right)\right] .
\end{aligned}
$$

Note $F(t)$ is increasing; then

$$
\begin{aligned}
& {\left[\varepsilon\left(F^{\prime}(\varepsilon)\right)^{2}+\frac{2 c}{2+p}\left(F^{2+p}(t)-F^{2+p}(\varepsilon)\right)\right]^{-1 / 2} F^{\prime}(t)} \\
& \quad \geq t^{-1 / 2} .
\end{aligned}
$$


Fix $\varepsilon>0$ and for $t>\varepsilon$ and integrate (34) on $[\varepsilon, t]$; it follows that

$$
\begin{aligned}
& \int_{F(\varepsilon)}^{F(t)}\left[\varepsilon\left(F^{\prime}(\varepsilon)\right)^{2}+\frac{2 c}{2+p}\left(y^{2+p}-F^{2+p}(\varepsilon)\right)\right]^{-1 / 2} d y \\
& \quad \geq 2\left(t^{1 / 2}-\varepsilon^{1 / 2}\right)>0 .
\end{aligned}
$$

Set $T_{1}=\int_{\alpha}^{+\infty}\left[\varepsilon\left(F^{\prime}(\varepsilon)\right)^{2}+(2 c /(2+p))\left(y^{2+p}-\right.\right.$ $\left.\left.F^{2+p}(\varepsilon)\right)\right]^{-1 / 2} d y$; then (35) implies that there exists some point $T<T_{1}$ such that

$$
\lim _{t \rightarrow T^{-}} F(t)=+\infty .
$$

Hence, there is $T \in\left(0, T_{1}\right)$ such that (30) holds for $b \in$ $(0,1 / 2)$. Then (10) and (11) are yielded by use of Hölder's inequality.

Finally, we complete the proof of Theorem 2.

\section{Singular Initial Value Problem}

In this section we show the blow-up of solution of singular initial value problem (1)-(2) with the idea of proof is similar to that used in Section 2, but it is more complicated in calculus.

Let $\psi(x)$ denote the first eigenfunction of Dirichlet problem (6); then there is only one eigenfunction $\psi(x)>0$ satisfying (7). As shown in [24], set $S_{r}=\{x:|x|<r\}=\Omega$ in (6) and denote $\psi_{r}(x)$ as the eigenfunction corresponding to the first eigenvalue $\lambda_{r}$; then $\psi_{r}(x)$ is also the function of $|x|$ only and $\lambda_{r}$ decrease with $r$.

Assume that

(c) nonnegative functions $\varphi_{1}(x), \varphi_{2}(x)$ have support in $S_{R-\delta}$ for some positive constant $R$ and $\delta \in(0, R)$; there exists $x_{i} \in S_{R-\delta}, i=1,2$ such that $\varphi_{1}\left(x_{1}\right)>0$ and $\varphi_{2}\left(x_{2}\right)>0$;

(d) $\int_{S_{R-\delta}} \psi_{r}(x) \varphi_{1}(x) d x=\alpha>0, \int_{S_{R-\delta}} \psi_{r}(x) \varphi_{2}(x) d x=$ $\beta>0$.

Set

$$
(\phi(t, \cdot), u(t, \cdot))=\int_{\mathbb{R}^{n}} \phi(t, x) u(t, x) d x .
$$

Taking a similar procedure of the proof of (36) and (46) in [25] with the principle of superposition, we can obtain a solution of problem (1)-(2) which is the representation at a point $(t, x)$ as a solution of the homogeneous linear equation plus a Volterra integral (formed by confluent hypergeometric function; see [26]) of the inhomogeneous term against an appropriate kernel over the retrograde cone through $(t, x)$. Then we give the following definition which is similar to definition 3 in [23].

Definition 3. One says that a solution of problem (1)-(2) has a cone compact support property if (for any $R$ ) whenever $\varphi_{i}(x), i=1,2$ has support in $\left\{x \in \mathbb{R}^{n}|| x \mid<R\right\}, u(t, \cdot)$ has support in $\left\{x \in \mathbb{R}^{n}|| x \mid<R+\gamma(t)\right\}$ for all $t \in(0, T)$, where

$$
\gamma(t)=2 \sqrt{t}
$$

Now fix $r>R$ and the value will be given later. Let $t \leq$ $\gamma^{-1}(r-R)$; then $u(t, x)$ given in Definition 3 has support in $S_{R-\delta+\gamma(t)} \times\left\{t \leq \gamma^{-1}(r-R)\right\}$, which is contained in $S_{r-\delta} \times\{t \leq$ $\left.\gamma^{-1}(r-R)\right\}$.

Definition 4. The function $u(t, x)$ is called a weak solution of problem (1)-(2) which enjoys the cone compact support property, if $u(t, \cdot)$ and $t^{b} \partial_{t} u(t, \cdot)$ are continuous with respect to $t$, if u satisfies (2), and if for all $\phi: S_{R-\delta} \times(0, T) \longrightarrow R$ which are twice continuously differentiable and satisfies

$$
\begin{aligned}
& t\left(\phi(t, \cdot), \partial_{t} u(t, \cdot)\right)=\int_{0}^{t}\left[(1-b)\left(\phi(\tau, \cdot), \partial_{\tau} u(\tau, \cdot)\right)\right. \\
& \quad+\tau\left(\partial_{\tau} \phi(\tau, \cdot), \partial_{\tau} u(\tau, \cdot)\right)+(\Delta \phi(\tau, \cdot), u(\tau, \cdot)) \\
& \quad+(\phi(\tau, \cdot), f(u(\tau, \cdot)))] d \tau .
\end{aligned}
$$

Theorem 5. Assume that a weak solution of problem (1)-(2) with $\mu \geq \lambda$ in (4) under conditions (c)-(d) in the sense of Definition 4 exists; then there is some positive constant $T$ such that

$$
\begin{aligned}
& \lim _{t \rightarrow T^{-}}\|u(t, \cdot)\|_{L^{\infty}\left(\mathbb{R}^{n}\right)}=+\infty . \\
& \lim _{t \rightarrow T^{-}}\|u(t, \cdot)\|_{L^{q}\left(\mathbb{R}^{n}\right)}=+\infty, \quad q \in[1,+\infty) .
\end{aligned}
$$

Proof. Define a function $\Psi_{r}(x)$; that is,

$$
\Psi_{r}(x)= \begin{cases}\psi_{r}(x), & |x| \leq r-\frac{\delta}{2} \\ \psi_{0}(x), & r-\frac{\delta}{2} \leq|x| \leq r \\ 0, & |x| \geq r\end{cases}
$$

where $\psi_{r}$ is first eigenfunction of Dirichlet problem $(6) ; \psi_{0}$ is a twice continuously differentiable function of $\rho=|x|$ which satisfies $\psi_{0}^{i}(r-\delta / 2)=\psi_{r}^{i}(r-\delta / 2)$ and $\psi_{0}^{i}(r)=0$ for $i=0,1,2$. When $t<\gamma^{-1}(r-R)$, substitute $\phi(t, x)$ by $\Psi_{r}(t, x)$ in (39); then it is easy to verify that

$$
\begin{aligned}
& t\left(\psi_{r}(\cdot), \partial_{t} u(t, \cdot)\right)=\int_{0}^{t}\left[(1-b)\left(\psi_{r}(\cdot), \partial_{\tau} u(\tau, \cdot)\right)\right. \\
& \left.\quad+\left(\Delta \psi_{r}(\cdot), u(\tau, \cdot)\right)+\left(\psi_{r}(\cdot), f(u(\tau, \cdot))\right)\right] d \tau
\end{aligned}
$$

since the support of $u(t, x)$ is contained in $S_{r-\delta} \times\left[0, \gamma^{-1}(r-R)\right)$.

For $t \leq \gamma^{-1}(r-R)$, set

$$
F_{r}(t)=\left(\psi_{r}(x), u(t, x)\right)=\int_{R^{n}} \psi_{r}(x) u(t, x) d x,
$$

and then, by the assumption,

$$
\begin{aligned}
F_{r}(t) & =\int_{B_{r-\delta}} \psi_{r}(x) u(t, x) d x, \\
F_{r}^{\prime}(t) & =\left(\psi_{r}(x), \partial_{t} u(t, x)\right) \\
& =\int_{B_{r-\delta}} \psi_{r}(x) \partial_{t} u(t, x) d x .
\end{aligned}
$$


In terms of (43) and (46), it follows that

$$
\begin{aligned}
& t F_{r}^{\prime}(t)=\int_{0}^{t}\left[(1-b)\left(\psi_{r}(\cdot), \partial_{\tau} u(\tau, \cdot)\right)\right. \\
& \left.\quad+\left(\Delta \psi_{r}(\cdot), u(\tau, \cdot)\right)+\left(\psi_{r}(\cdot), f(u(\tau, \cdot))\right)\right] d \tau .
\end{aligned}
$$

Therefore, we obtain

$$
\begin{gathered}
F_{r}^{\prime \prime}(t)=\left(t^{-1}\left(t F_{r}^{\prime}(t)\right)\right)^{\prime}=t^{-1}\left[-b\left(\psi_{r}(x), \partial_{t} u(t, x)\right)\right. \\
\left.+\left(\psi_{r}(x), f(u(t, x))\right)-\lambda_{r}\left(\psi_{r}(x), u(t, x)\right)\right]
\end{gathered}
$$

which is equivalent to

$$
\begin{aligned}
& \frac{d}{d t}\left(t^{b} F^{\prime}(t)\right) \\
& \geq c t^{b-1}\left(\left(\psi_{r}(x), f(u(t, x))\right)-\lambda_{r}\left(\psi_{r}(x), u(t, x)\right)\right) .
\end{aligned}
$$

In terms of the convexity of $f(\cdot)$ and Jensen's inequality, one has

$$
\begin{gathered}
\left(\psi_{r}(x), f(u(t, x))=\int_{R^{n}} \psi_{r}(x) f(u(t, x)) d x\right. \\
\left.\geq f\left(\int_{R^{n}} \psi_{r}(x) u(t, x) d x\right)\right)=f\left(F_{r}(t)\right) .
\end{gathered}
$$

Then substituting (50) into (49), together with (4) for $\lambda_{r}=\mu$, we obtain

$$
\frac{d}{d t}\left(t^{b} F^{\prime}(t)\right) \geq c t^{b-1} F_{r}^{1+p}(t)
$$

In fact, if set $f(s)=s^{1+p}$, then we can choose $R$ such that $\lambda_{r}<\alpha^{p}$ for Dirichlet problem (6) in $S_{r}$, and then (51) holds which is independent of (4).

Note that $F_{r}(0)=\alpha>0$ and $\lim _{t \rightarrow 0} t^{b} F_{r}^{\prime}(0)=\beta>0$; then there exists an interval $(0, \eta)$ such that $F_{r}(t)>\alpha$ for $t \in(0, \eta)$. Hence, on $(0, \eta)$,

$$
\frac{d}{d t}\left(t^{b} F_{r}^{\prime}(t)\right) \geq c t^{b-1} F_{r}^{1+p}(t)>0 .
$$

This means that $t^{b} F_{r}^{\prime}(t)$ is strictly increasing on $(0, \eta)$. Then we obtain $F_{r}^{\prime}(t)>\beta t^{-b}>0$ on $(0, \eta)$. Multiply $t^{b} F_{r}^{\prime}(t)$ on (51) and then integrate it over $(\varepsilon, t)$ where $(\varepsilon, t) \in(0, \eta)$ :

$$
\begin{aligned}
\left(t^{b} F_{r}^{\prime}(t)\right)^{2} \geq & \left(\varepsilon^{b} F_{r}^{\prime}(\varepsilon)\right)^{2} \\
& +2 c \int_{\varepsilon}^{t} \tau^{2 b-1} F_{r}^{1+p}(\tau) F_{r}^{\prime}(\tau) d \tau .
\end{aligned}
$$

It follows that $F_{r}^{\prime}(t)>\beta t^{-b}$ and $F_{r}(t)>\alpha$ on the entire existence interval.

Now, for $\varepsilon>0$, we set

$$
\begin{aligned}
& H_{r}(t)=\int_{\varepsilon}^{t} \tau^{2 b-1} F_{r}^{1+p}(\tau) F_{r}^{\prime}(\tau) d \tau, \\
& G_{r}(t)=(2+p)^{-1} F_{r}^{2+p}(t) .
\end{aligned}
$$

Then, there, $H_{r}^{\prime}(t)=t^{2 b-1} G_{r}^{\prime}(t)$ holds and $H_{r}(t)>0, G_{r}(t)>$ 0 . For $b \in[1 / 2,1)$, integrating by parts, it follows that $H_{r}(t)$

$$
\begin{aligned}
& \leq t^{2 b-1} G_{r}(t) \\
& \quad-\left(\varepsilon^{2 b-1} G_{r}(\varepsilon)+(2 b-1) \int_{\varepsilon}^{t} \tau^{2(b-1)} G_{r}(\tau) d \tau\right) \\
& \leq t^{2 b-1} G_{r}(t) .
\end{aligned}
$$

Combining to (51)-(56), we derive that

$$
\begin{aligned}
& \left(H_{r}^{\prime}(t)\right)^{2} \geq(2+p)^{2+2 p} \\
& \cdot t^{-1+p(1-2 b) /(2+p)}\left(\left(\varepsilon^{b} F_{r}^{\prime}(\varepsilon)\right)^{2}+2 c H_{r}(t)\right) \\
& \cdot H_{r}^{1+p /(2+p)}(t),
\end{aligned}
$$

which is equivalent to

$$
\begin{aligned}
& {\left[\left(\left(\varepsilon^{b} F_{r}^{\prime}(\varepsilon)\right)^{2}+2 c H(t)\right) H_{r}^{1+p /(2+p)}(t)\right]^{-1 / 2} H_{r}^{\prime}(t)} \\
& \quad \geq(2+p)^{1+p} t^{-1 / 2+p(1-2 b) / 2(2+p)} .
\end{aligned}
$$

Then, for $b \in[1 / 2,1)$, integrating $(58)$ over $(\varepsilon, t)$, we obtain

$$
\begin{aligned}
& \int_{H_{r}(\varepsilon)}^{H_{r}(t)}\left(\left(\varepsilon^{b} F_{r}^{\prime}(\varepsilon)\right)^{2}+2 c H_{r}(\tau)\right)^{-1 / 2} \\
& \quad \cdot H_{r}^{-1 / 2-p / 2(2+p)}(\tau) d H_{r}(\tau) \\
& \quad \geq \int_{\varepsilon}^{t}(2+p)^{1+p} \tau^{-1 / 2+p(1-2 b) / 2(2+p)} d \tau \geq(2+p)^{1+p} \\
& \quad \cdot\left(t^{1 / 2+p(1-2 b) / 2(2+p)}\right. \\
& \left.\quad-\varepsilon^{1 / 2+p(1-2 b) / 2(2+p)}\right) .
\end{aligned}
$$

According to the previous analysis, we have $\varepsilon^{b} F_{r}^{\prime}(\varepsilon)>\beta>0$ independent of $r$; then

$$
\begin{aligned}
& \int_{H_{r}(\varepsilon)}^{H_{r}(t)}\left(\left(\varepsilon^{b} F_{r}^{\prime}(\varepsilon)\right)^{2}+2 c H_{r}(\tau)\right)^{-1 / 2} \\
& \cdot H_{r}^{-1 / 2-p / 2(2+p)}(\tau) d H_{r}(\tau) \\
& \quad \leq \int_{H_{r}(\varepsilon)}^{H_{r}(t)}\left(\left(\varepsilon^{b} F_{r}^{\prime}(\varepsilon)\right)^{2}+2 c \tau\right)^{-1 / 2} \tau^{-1 / 2-p / 2(2+p)} d \tau \\
& <\int_{\beta}^{+\infty}\left(\left(\varepsilon^{b} F_{r}^{\prime}(\varepsilon)\right)^{2}+2 c t\right)^{-1 / 2} t^{-1 / 2-p / 2(2+p)} d t .
\end{aligned}
$$

Set $K_{0}=\int_{\beta}^{+\infty}(2 c t)^{-1 / 2} t^{-1 / 2-p / 2(2+p)} d t$, in terms of (59)-(60); then $K_{0}$ is a finite positive number independent of $r$ and $\varepsilon$, and

$$
\begin{aligned}
& (2+p)^{1+p}\left(t^{1 / 2+p(1-2 b) / 2(2+p)}-\varepsilon^{1 / 2+p(1-2 b) / 2(2+p)}\right) \\
& \quad<K_{0} .
\end{aligned}
$$


Now fix $\varepsilon$; we choose $r=r_{0}$,

$$
\begin{aligned}
r_{0} & =\left(K_{0}(2+p)^{-1-p}\right. \\
& \left.+\varepsilon^{1 / 2+p(1-2 b) / 2(2+p)}\right)^{1 /(1 / 2+p(1-2 b) / 2(2+p))}+2 R,
\end{aligned}
$$

and then we obtain

$$
t<r_{0}-2 R<r_{0}-R
$$

for all $t \in(\varepsilon, \eta) \cap\left(0, r_{0}-R\right)$, where $(0, \eta)$ is the existence interval of solution (1)-(2). Hence there is a $T \leq r_{0}-2 R$, such that

$$
\lim _{t \longrightarrow T^{-}} H_{r_{0}}(t)=+\infty
$$

From (55)-(56) and (64), we obtain

$$
\lim _{t \longrightarrow T^{-}} F_{r_{0}}(t)=+\infty
$$

At last, by use of Hölder inequality on (65), we derive (40) and (41) for $b \in[1 / 2,1)$.

For $b \in(0,1 / 2)$, we also obtain (35) with the term $F$ being substituted by $F_{r}$ by a procedure as mentioned in Section 2; that is,

$$
\begin{aligned}
& \int_{F_{r}(\varepsilon)}^{F_{r}(t)}\left[\varepsilon\left(F_{r}^{\prime}(\varepsilon)\right)^{2}+\frac{2 c}{2+p}\left(y^{2+p}-F_{r}^{2+p}(\varepsilon)\right)\right]^{-1 / 2} d y \\
& \quad \geq 2\left(t^{1 / 2}-\varepsilon^{1 / 2}\right)>0
\end{aligned}
$$

Since we do not know the sign of the term $\varepsilon\left(F_{r}^{\prime}(\varepsilon)\right)^{2}-(2 c /(2+$ p)) $F_{r}^{2+p}(\varepsilon)$, we calculate

$$
\begin{aligned}
& \int_{F_{r}(\varepsilon)}^{F_{r}(t)}\left[\varepsilon\left(F_{r}^{\prime}(\varepsilon)\right)^{2}+\frac{2 c}{2+p}\left(y^{2+p}-F_{r}^{2+p}(\varepsilon)\right)\right]^{-1 / 2} d y \\
& \quad \leq\left(\frac{2 c}{2+p}\right)^{-1 / 2} \int_{F_{r}(\varepsilon)}^{+\infty}\left(y^{2+p}-F_{r}^{2+p}(\varepsilon)\right)^{-1 / 2} d y \\
& \quad=\left(\frac{2 c}{2+p}\right)^{-1 / 2} F_{r}^{-p / 2}(\varepsilon) \int_{1}^{+\infty}\left(z^{2+p}-1\right)^{-1 / 2} d z \\
& \quad<\left(\frac{2 c}{2+p}\right)^{-1 / 2} \alpha^{-p / 2} \int_{1}^{+\infty}\left(z^{2+p}-1\right)^{-1 / 2} d z \\
& <+\infty
\end{aligned}
$$

where we use $p>0$ and $F_{r}(\varepsilon)>\alpha$.

Let $K_{1}:=(2 c /(2+p))^{-1 / 2} \alpha^{-p / 2} \int_{1}^{+\infty}\left(z^{2+p}-1\right)^{-1 / 2} d z$ which is a finite positive constant independent of $r$ and $\varepsilon$; then

$$
K_{1}>2\left(t^{1 / 2}-\varepsilon^{1 / 2}\right) .
$$

Fixing $\varepsilon$, we choose $r=r_{1}$ :

$$
r_{1}=\left(\varepsilon^{1 / 2}+\frac{1}{2} K_{1}\right)^{2}+2 R
$$

and then it follows from (68) that

$$
t<r_{1}-2 R<r_{1}-R
$$

for all $t \in(\varepsilon, \eta) \cap\left(0, r_{1}-R\right)$, where $(0, \eta)$ is the existence interval of solution (1)-(2). Hence there is $T \leq r_{1}-2 R$, such that

$$
\lim _{t \rightarrow T^{-}} H_{r_{1}}(t)=+\infty \text {. }
$$

By a computation with (55)-(56) and (71), we obtain

$$
\lim _{t \rightarrow T^{-}} F_{r_{1}}(t)=+\infty .
$$

Hence, in terms of Hölder inequality, the last formula implies (40) and (41) for $b \in(0,1 / 2)$.

Then we conclude that Theorem 5 holds.

\section{Data Availability}

No data were used to support this study.

\section{Conflicts of Interest}

The author declares that there are no conflicts of interest regarding the publication of this paper.

\section{Acknowledgments}

This work was supported by NNSF of China (Grant no. 11326152), NSF of Jiangsu Province of China (Grant no. BK20130736), and NSF of Nanjing Institute of Technology (CKJB201709).

\section{References}

[1] T. Hristov, "Singular solutions to Protter problem for Keldysh type equations," in Proceedings of the 40th International Conference of Applications of Mathematics in Engineering and Economics, AMEE 2014, pp. 255-262, Bulgaria, June 2014.

[2] T. H. Otway, The Dirichlet Problem for Elliptic-Hyperbolic Equations of Keldysh Type, vol. 2043 of Lecture Notes in Mathematics, Springer, Berlin, Germany, 2012.

[3] S. Chen, "Mixed type equations in gas dynamics," Quarterly of Applied Mathematics, vol. 68, no. 3, pp. 487-511, 2010.

[4] C. S. Morawetz, "Mixed equations and transonic flow," Journal of Hyperbolic Differential Equations, vol. 1, no. 1, pp. 1-26, 2004.

[5] S. Chen, "The fundamental solution of the Keldysh type operator," Science China Mathematics, vol. 52, no. 9, pp. 1829-1843, 2009.

[6] Nedyu Popivanov, Tsvetan Hristov, Aleksey Nikolov, and Manfred Schneider, "Singular Solutions to a $(3+1)$-D ProtterMorawetz Problem for Keldysh-Type Equations," Advances in Mathematical Physics, vol. 2017, Article ID 1571959, 16 pages, 2017.

[7] N. Popivanov, T. Hristov, A. Nikolov, and M. Schneider, "On the existence and uniqueness of a generalized solution of the Protter problem for $(3+1)$-D Keldysh-type equations," Boundary Value Problems, vol. 2017, no. 26, 2017.

[8] P. Brenner and W. von Wahl, "Global classical solutions of nonlinear wave equations," Mathematische Zeitschrift, vol. 176, no. 1, pp. 87-121, 1981. 
[9] M. G. Grillakis, "Regularity and asymptotic behaviour of the wave equation with a critical nonlinearity," Annals of Mathematics: Second Series, vol. 132, no. 3, pp. 485-509, 1990.

[10] V. Georgiev, H. Lindblad, and C. . Sogge, "Weighted Strichartz estimates and global existence for semilinear wave equations," American Journal of Mathematics, vol. 119, no. 6, pp. 1291-1319, 1997.

[11] K. Jörgens, "Das Anfangswertproblem in Grossen fur eine klasse nichtlinearer Wellengleichungen," Mathematische Zeitschrift, vol. 77, no. 1, pp. 295-308, 1961.

[12] J. B. Keller, “On solutions of nonlinear wave equations," Communications on Pure and Applied Mathematics, vol. 10, pp. 523530, 1957.

[13] K. Jörgens, "Nonlinear wave equations," in Nonlinear Wave Equations, vol. 2, University of Colorado, Department of Mathematics, Denver, Colo, USA, 2017.

[14] R. T. Glassey, "Blow-up theorems for nonlinear wave equations," Mathematische Zeitschrift, vol. 132, pp. 183-203, 1973.

[15] X. Liu and Y. Zhou, "Global nonexistence of solutions to a semilinear wave equation in the Minkowski space," Applied Mathematics Letters, vol. 21, no. 8, pp. 849-854, 2008.

[16] S. A. Messaoudi, "Blow up in a nonlinearly damped wave equation," Mathematische Nachrichten, vol. 231, no. 1, pp. 1-7, 2001.

[17] T. C. Sideris, "Nonexistence of global solutions to semilinear wave equations in high dimensions," Journal of Differential Equations, vol. 52, no. 3, pp. 378-406, 1984.

[18] B. T. Yordanov and Q. S. Zhang, "Finite time blow up for critical wave equations in high dimensions," Journal of Functional Analysis, vol. 231, no. 2, pp. 361-374, 2006.

[19] S. Kaplan, "On the growth of solutions of quasi-linear parabolic equations," Communications on Pure and Applied Mathematics, vol. 16, pp. 305-330, 1963.

[20] H. Fujita, "On the blowing up of solutions of the Cauchy problem for $\partial \mathrm{tu}=\Delta \mathrm{u}+\mathrm{u} 1+\alpha[\mathrm{J}]$," Journal of the Faculty of Science, vol. 13, 1966.

[21] H. Fujita, "On some nonexistence and nonuniqueness theorems for nonlinear parabolic equations," Proceedings of Symposia in Pure Mathematics, vol. 18, pp. 105-113, 1970.

[22] H. A. Levine, "On the nonexistence of global solutions to a nonlinear Euler-Poisson-Darboux equation," Journal of Mathematical Analysis and Applications, vol. 48, pp. 646-651, 1974.

[23] H. A. Levine, "Growth of solutions of generalized nonlinear Euler-Poisson-Darboux equations," Archive for Rational Mechanics and Analysis, vol. 61, no. 1, pp. 77-89, 1976.

[24] R. Courant and D. Hilbert, Methods of Mathematical Physics, vol. 1, CUP Archive, 1966.

[25] K. Zhang, "The Cauchy problem for semilinear hyperbolic equation with characteristic degeneration on the initial hyperplane," Mathematical Methods in the Applied Sciences, vol. 41, no. 6, pp. 2429-2441, 2018.

[26] A. Erdlyi, W. Magnus, F. Oberhettinger et al., Higher Transcendental Functions, vol. 1, McGraw-Hill, New York, NY, USA, 1953. 


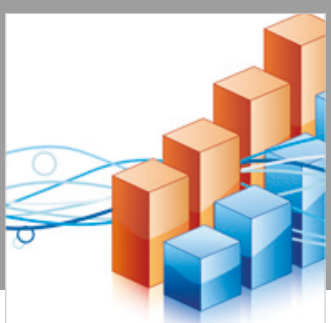

Advances in

Operations Research

\section{-n-m}
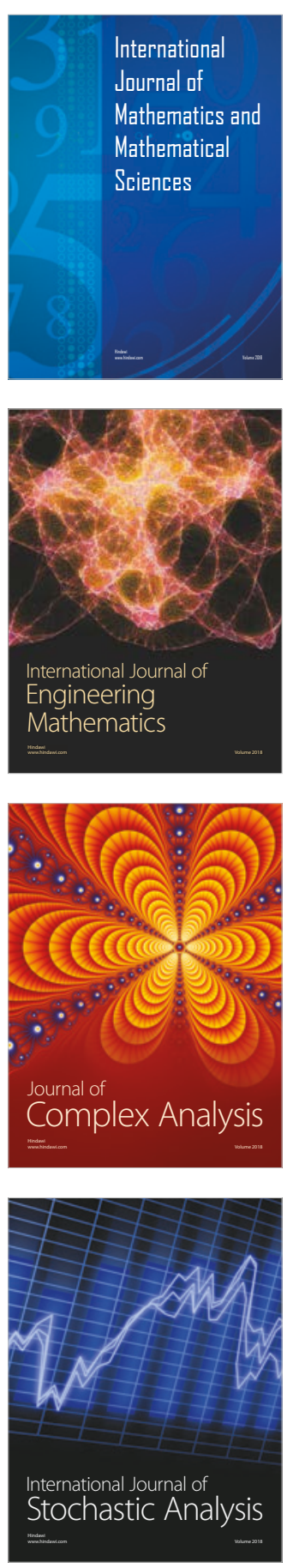
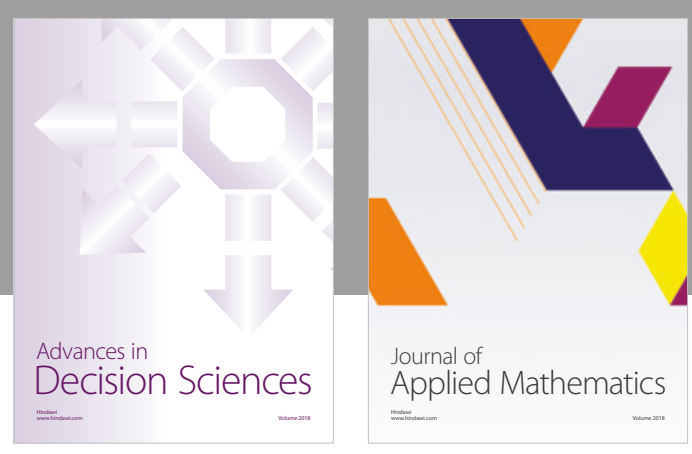

Journal of

Applied Mathematics
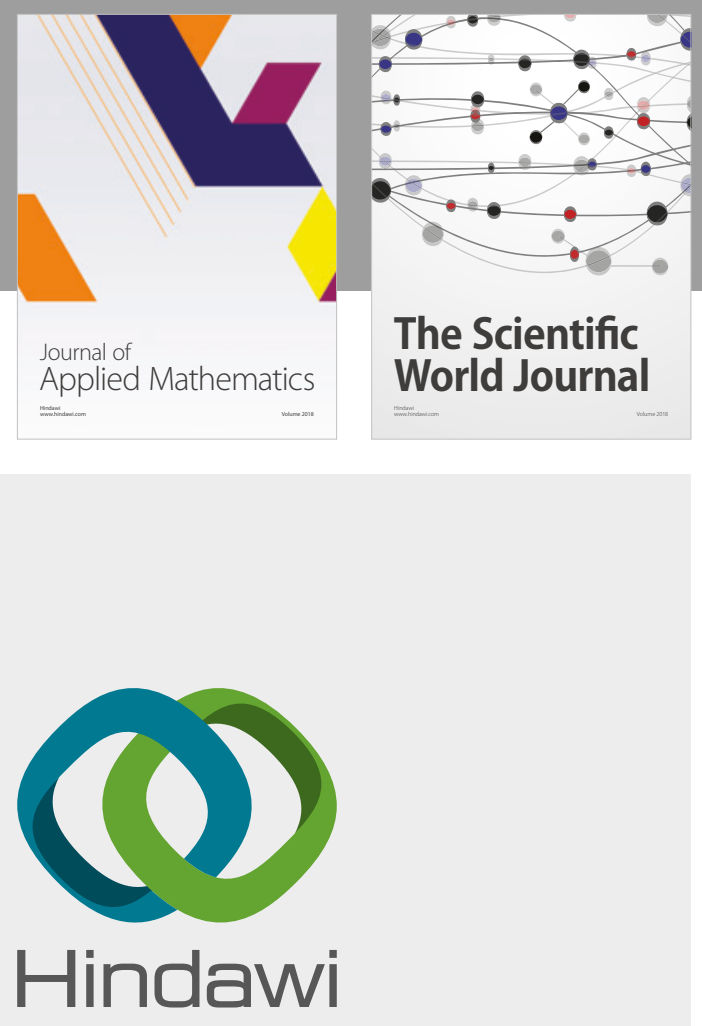

Submit your manuscripts at

www.hindawi.com

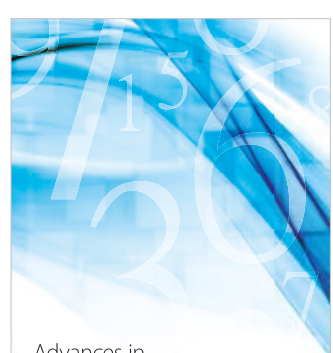

Advances in
Numerical Analysis
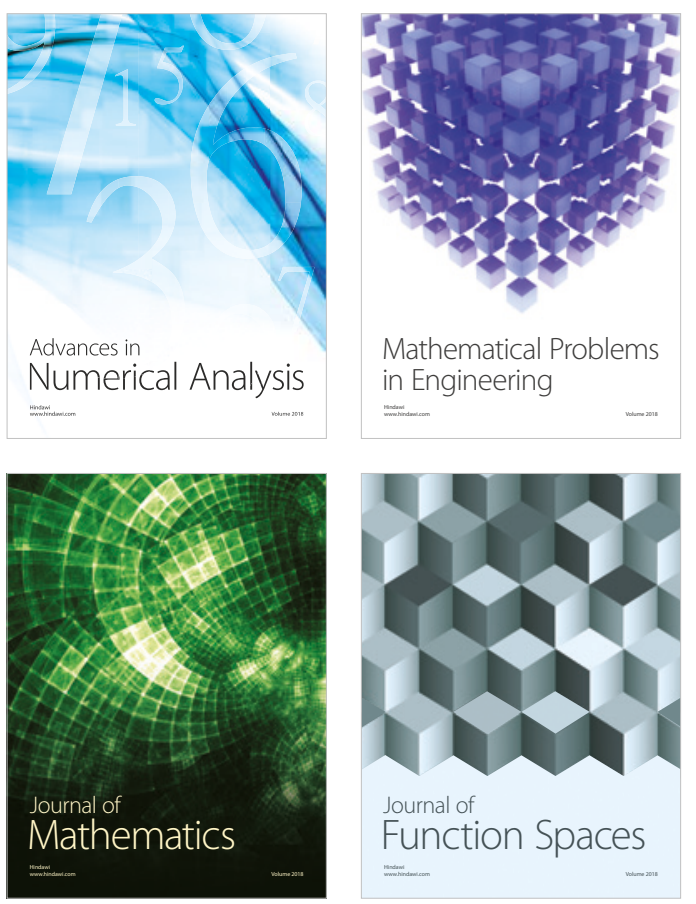

Mathematical Problems in Engineering

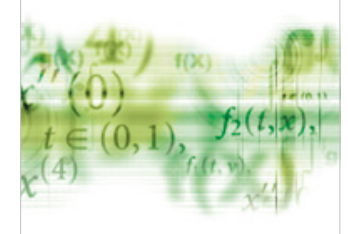

International Journal of

Differential Equations

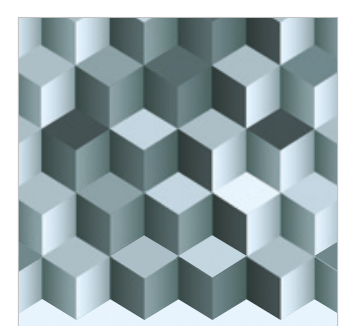

Journal of

Function Spaces

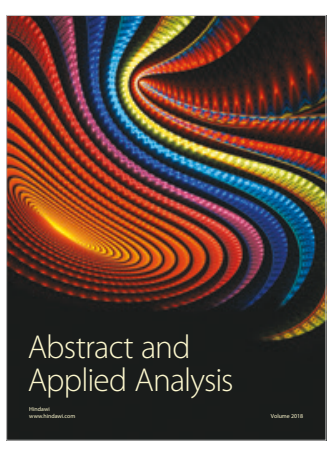

The Scientific

World Journal

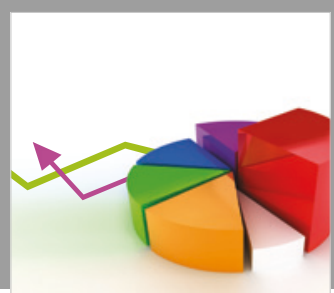

Journal of

Probability and Statistics
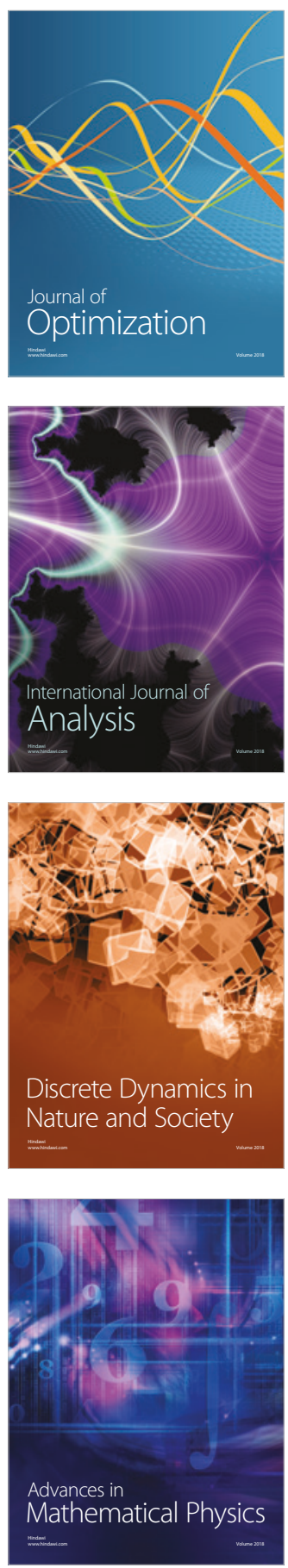